\title{
Multiple Mechanisms for Integrating Proprioceptive Inputs That Converge on the Same Motor Pattern-Generating Network
}

\author{
Gregory Barrière, John Simmers, and Denis Combes \\ Université de Bordeaux, Laboratoire Mouvement Adaptation Cognition, Unité Mixte de Recherche 5227, Centre National de la Recherche Scientifique, \\ Bordeaux 33076, France
}

\begin{abstract}
Movement-derived sensory feedback adapts centrally generated motor programs to changing behavioral demands. Motor circuit output may also be shaped by distinct proprioceptive systems with different central actions, although little is known about the integrative processes by which such convergent sensorimotor regulation occurs. Here, we explore the combined actions of two previously identified proprioceptors on the gastric mill motor network in the lobster stomatogastric nervous system. Both mechanoreceptors [anterior gastric receptor (AGR) and posterior stomach receptor (PSR)] access the gastric circuit via the same pair of identified projection interneurons that either excite [commissural gastric (CG)] or inhibit [gastric inhibitor (GI)] different subsets of gastric network neurons. Mechanosensory information from the two receptors is integrated upstream to the gastric circuit at two levels: (1) postsynaptically, where both receptors excite the GI neuron while exerting opposing effects on the CG neuron, and (2) presynaptically, where PSR reduces AGR's excitation of the CG projection neuron. Concomitantly PSR selectively enhances AGR's activation of the GI neuron, possibly also via a presynaptic action. PSR's influences also far outlast its transient synaptic effects, indicating the additional involvement of modulatory processes. Consequently, PSR activation causes parallel input from AGR to be conveyed preferentially via the GI interneuron, resulting in a prolonged switch in the pattern of gastric circuit output. Therefore, via a combination of short- and long-lasting, presynaptic and postsynaptic actions, one proprioceptive system is able to promote its impact on a target motor network by biasing the access of a different sensory system to the same circuit.
\end{abstract}

Key words: stomatogastric system; gastric mill network; proprioceptor; sensory convergence; projection neurons; circuit reconfiguration

\section{Introduction}

Sensory inputs play a major role in defining the motor output patterns responsible for most behavioral tasks. Multiple studies on both invertebrate and vertebrate motor systems have shown that centrally generated motor patterns can be initiated, terminated, entrained, or reconfigured by specific sensory commands (Katz and Harris-Warrick, 1990; Pearson, 1995; Soffe, 1997; Combes et al., 1999b; Grillner and Wallén, 2002; Blitz et al., 2004; Büschges, 2005; Yakovenko et al., 2005; Rossignol et al., 2006). Most central pattern generators (CPGs), the neural networks that generate rhythmic behaviors such as locomotion, respiration, and feeding-related acts, are also subjected to a variety of sensory influences. The vertebrate locomotor CPG, for example, receives convergent sensory information from muscle spindles, tendon organs, and cutaneous receptors (Lundberg et al., 1978; Hultborn, 2006; Hultborn and Nielsen, 2007). Such multiple afferent

Received May 7, 2008; revised June 30, 2008; accepted July 23, 2008.

Correspondence should be addressed to Dr. Denis Combes, Laboratoire Mouvement Adaptation Cognition, Unité Mixte de Recherche 5227, Centre National de la Recherche Scientifique, Bâtiment 2A, Université de Bordeaux, 146 rue Léo Saignat, Bordeaux 33076, France. E-mail: denis.combes@u-bordeaux2.fr.

G. Barrière's present address: Centre de Recherche en Sciences Neurologiques, Faculté de Médecine, Université de Montréal, C.P. 6128, Succursale Centre-ville, Montréal, Canada H3C 3J7.

DOI:10.1523/JNEUROSCI.2095-08.2008

Copyright $\odot 2008$ Society for Neuroscience $\quad 0270-6474 / 08 / 288810-11 \$ 15.00 / 0$ cues, whether arising from distinct proprioceptor systems or from different sensory modalities (mechanosensory vs visual signaling, for example), must be integrated either directly by the target CPG itself or at a precircuit level to produce adaptive motor responses (Jankowska and McCrea, 1983; Blitz et al., 2004; Hultborn, 2006).

Although the ability of individual sensory inputs to regulate motor circuit output has been intensively studied, the cellular and synaptic processes by which converging afferent signals interact to shape motor output is still poorly understood. Invertebrate preparations, because of their accessibility for electrophysiological investigation and their relative simplicity, have contributed significantly to our understanding of the mechanisms underlying the sensory regulation of CPG output (Katz et al., 1989; Harris-Warrick and Katz, 1990; Hooper et al., 1990; Nargeot and Moulins, 1997; Combes et al., 1999b; Beenhakker et al., 2004; Büschges, 2005). In the present study, we have used the stomatogastric nervous system (STNS) of the lobster Homarus gammarus to study the integrative interactions between two identified sensory systems that converge onto the well known gastric mill CPG in the stomatogastric ganglion (STG). These input pathways arise from separate stretch receptor organs, the single neuron anterior gastric receptor (AGR) (Simmers and Moulins, 1988a; Combes et al., 1995, 1999a) and the multicellular posterior 

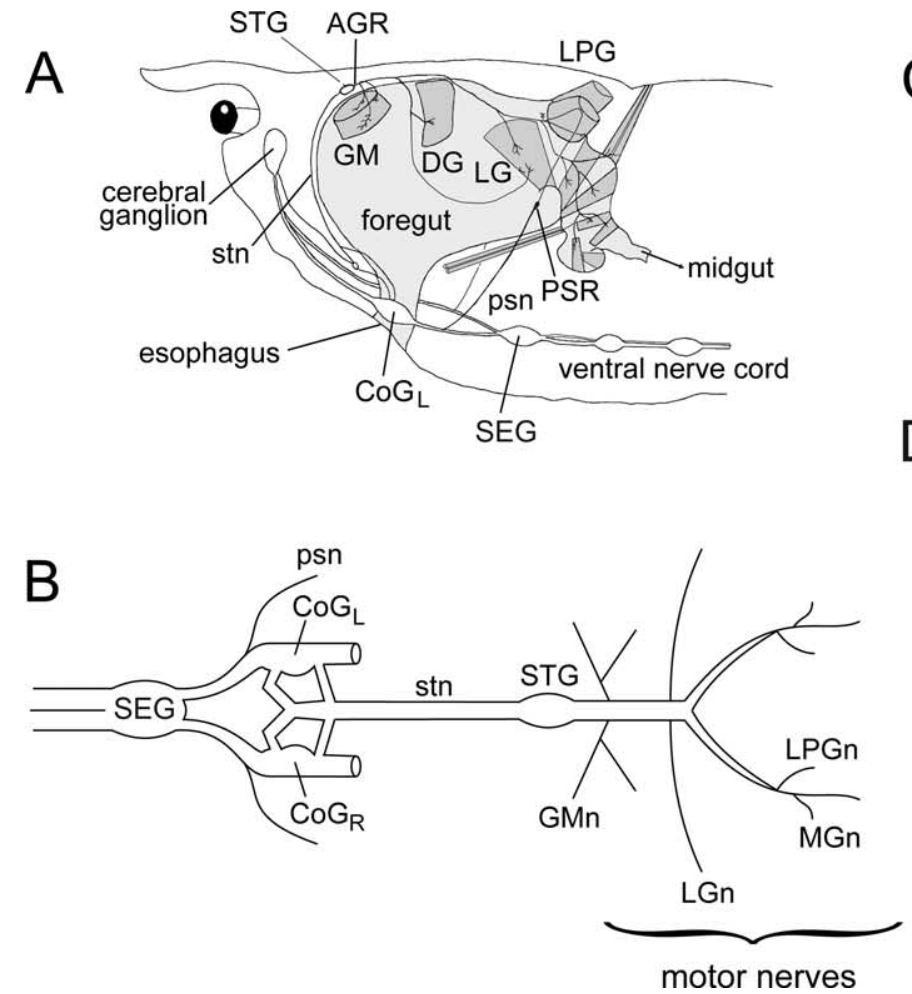

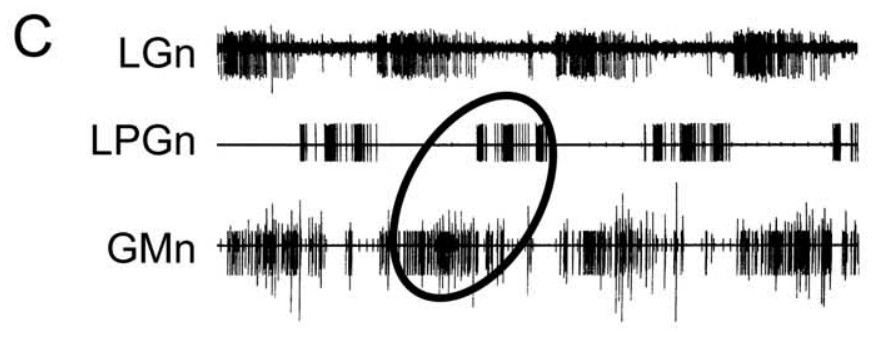

D

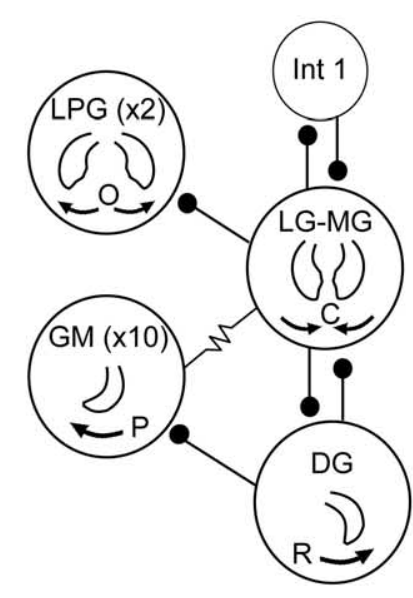

\section{gastric motor network}

Figure 1. The STNS and gastric mill network of the lobster Homarus gammarus. $\boldsymbol{A}$, Lateral view showing location of the foregut, gastric mill muscles, and STNS in situ. $\boldsymbol{B}$, Isolated STNS in vitro. The gastric mill motor circuit, including the GM, LG-MG, DG, and LPG motoneurons, is located in the STG, from which their axons project caudally in separate bilateral nerve branches. The STG is connected to the two CoGs and the SEG via the single stn. C, Typical gastric mill output pattern recorded extracellularly from indicated gastric motor nerves (n) in an isolated STNS. GM motoneurons fire in antiphase with LPG motoneurons and in phase with the LG motoneuron. D, Synaptic connectivity of the gastric mill network. Stick and ball symbols, Chemical inhibitory synapses; resistor symbol, electrical coupling. Numbers of neurons of each type are indicated in parentheses, when involving more than a single neuron. Neurons of each functional group are electrically coupled, including the LG-MG neurons. Gastric teeth movements driven by each neuron type is also indicated. GM and DG motoneurons are responsible for protraction (P) and retraction ( $R$ ) of the medial tooth, whereas

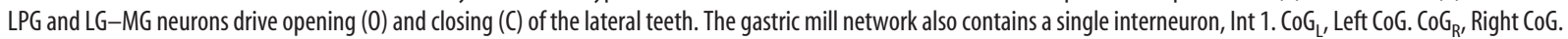

stomach receptor (PSR) (Dando and Laverack, 1969). AGR and PSR are activated by the contraction of different gastric muscles in vivo to provide movement-related feedback to the gastric CPG. Here, we show that the convergent mechanosensory information arising from these two receptors is integrated at two cellular loci before reaching the gastric mill circuit: postsynaptically by acting on the same second-order interneurons intercalated between the receptors and the gastric motor network, and presynaptically via direct interactions between the central projections of the receptors themselves. Together, these multiple upstream actions enable one proprioceptor (PSR) to control the influence of the other proprioceptor (AGR) on gastric motor output by selecting the projection pathway through which AGR accesses the gastric mill circuit and thereby the output pattern the network expresses.

\section{Materials and Methods}

All experiments were performed on adult European rock lobsters, Homarus gammarus, purchased from local commercial suppliers and kept in aquaria with fresh running sea water maintained at $16^{\circ} \mathrm{C}$. Before dissection, animals were cold-anesthetized in ice for $30 \mathrm{~min}$, and the STNS was then isolated from the foregut (Fig. 1A). The bilateral dendritic receptor endings of AGR were dissected from the two tendons of the anterior gastric muscles (gm1) as previously described (Combes et al., 1993). Similarly, each of the bilateral posterior stomach nerves (psn) that carries axons from the PSR of each side was located at its entry to the corresponding circumesophageal connective posterior to the left or right commissural ganglion (Fig. 1A). The peripheral projections of each psn, which sometimes included the distally located somata of the PSR itself (see Fig. 3), were then carefully dissected free from the internal lateral wall of the thorax (Dando and Laverack, 1969). Motor nerve branches containing the axons of individual motoneuron types [the lateral posterior gastric (LPG), gastric mill (GM), and lateral gastric (LG) motoneurons] were obtained by dissecting the distal gastric mill innervation to the corresponding target muscle. The entire STNS preparation, including the STG, the two bilateral commissural ganglia (CoGs), the single subesophageal ganglion (SEG), and their interconnecting nerves (Fig. 1B), was then transferred and pinned down on a silicon elastomer-lined (Sylgard 184; Dow Corning) Petri dish. The preparation was superfused continuously with aerated lobster saline maintained at $15-18^{\circ} \mathrm{C}$ with a thermoelectric cooling system (Dagan Corporation). The saline composition was as follows (in $\mathrm{mm}$ ): $479.12 \mathrm{NaCl}, 12.74 \mathrm{KCl}, 13.67 \mathrm{CaCl}_{2}$ $2 \mathrm{H}_{2} \mathrm{O}, 10 \mathrm{MgSO}_{4}, 3.91 \mathrm{Na}_{2} \mathrm{SO}_{4}$, and 5 HEPES, buffered to $\mathrm{pH}$ 7.45. In some experiments, the normal bathing saline was replaced with a high $(2.5 \times$ normal $)$ divalent cation solution (i.e., with $34.25 \mathrm{~mm} \mathrm{Ca}^{2+}$ and 25 $\mathrm{mm} \mathrm{Mg}^{2+}$ ) to test for monosynaptic connectivity (Simmers and Moulins, 1988a).

Extracellular recordings were made with platinum wire electrodes placed against selected nerves and insulated electrically with Vaseline. Intracellular recordings were made with glass microelectrodes (resistance 10-30 M $\Omega$ filled with $3 \mathrm{~m} \mathrm{KAc}$ ) placed in the somata (AGR and STG gastric circuit neurons), dendrites [commissural gastric (CG) and gastric inhibitor (GI) interneurons], or axon terminals (AGR) after desheathing the appropriate ganglion. Motoneurons were identified by correlating intrasomatic spikes with action potentials recorded en passant in terminal motor nerve branches (see above). After impalement, the CG and GI projection neurons were identified as described previously (Combes et al., 1999a). AGR was recorded intracellularly from its soma, which lies immediately posterior to the STG (see Fig. 3) or from the receptor's axon close to its terminals in either CoG. The axons of the PSR organs were recorded/stimulated extracellularly with Vaseline-insulated wire electrodes placed against either the left or right psn. 
AGR was stained by iontophoretic injection (with 5-10 nA negative current pulses for 20 min) of 3\% 5-6 carboxyfluorescein (Sigma) via the intrasomatic recording electrode. The sensory neuron was then visualized with blue light illumination (wavelength, $450-488 \mathrm{~nm}$ ). PSR somata and central axonal projections were labeled, respectively, by passive retrograde or anterograde migration of rhodamine-dextran ( $5 \%$ in 2 м KAc; Sigma) after cutting the psn either distally close to the receptors' somata or near the entry of its axons into the circumesophageal connective. Dye migration proceeded for $24 \mathrm{~h}$ at $4^{\circ} \mathrm{C}$, and preparations were then dehydrated, cleared in methyl salicylate, and visualized under green light (wavelength, $547 \mathrm{~nm})$.

All electrophysiological phenomena reported in this study were observed in at least three different experiments, which in each case represents a minimum of $80 \%$ of tested preparations. In experiments in which the phase of gastric motoneuron activity in motor patterns elicited by rhythmic AGR stimulation was determined (see Figs. 5, 7), the onset times of successive bursts in the LPG and GM neurons were expressed as a fraction of the corresponding AGR-elicited cycle that had elapsed before the start of a given motor burst. In other experiments (see Fig. 9), the amplitudes of AGRevoked EPSPs in the GI neuron that occurred at post-psn stimulus intervals of 1,20 , and $100 \mathrm{~s}$ were compared with control (prestimulus) amplitudes by repeated-measures ANOVA followed by the Dunnett's posttest performed with GraphPad Prism software for Windows version 4. All averaged data are given as mean $\pm \mathrm{SD}$, and statistical significance was set at a probability level of 0.05 .

\section{Results}

The gastric mill network and its capacity to switch between different motor output patterns

The gastric motor circuit in the STG of H. gammarus consists of four motoneuron subsets (Fig. 1D), of which two control protraction (10 GM neurons) and retraction [1 dorsal gastric (DG) neuron] of the gastric medial tooth, whereas the remaining two drive opening (2 LPG neurons) and closing [the single LG and medial gastric (MG) neurons] of the lateral teeth (Selverston and Moulins, 1987; Combes et al., 1999b). This network, which also includes a single interneuron (Int1), is spontaneously active in the isolated STNS, producing a stereotyped motor pattern (Fig. $1 C)$ that consists of coincident bursting in the electrically coupled LG-MG and GM motoneurons (which innervate the lateral and medial teeth power-stroke muscles, respectively) occurring in phase opposition with bursts in the LPG and DG motoneurons (that innervate the antagonistic lateral and medial teeth returnstroke muscles, respectively).

As previously described (Combes et al., 1999a), the gastric mill circuit receives input from two bilateral pairs of identified premotor interneurons that originate in the more rostral CoGs. One of these projection neurons, the CG interneuron, makes direct excitatory synaptic connections with the LPG and GM gastric motoneurons (Fig. 2). Through a combination of this projection neuron excitation and the strong LG-MG-mediated inhibition of the LPG neurons, cyclic activation of a CG neuron elicits coactivation of the electrically coupled GM and LG-MG neurons in
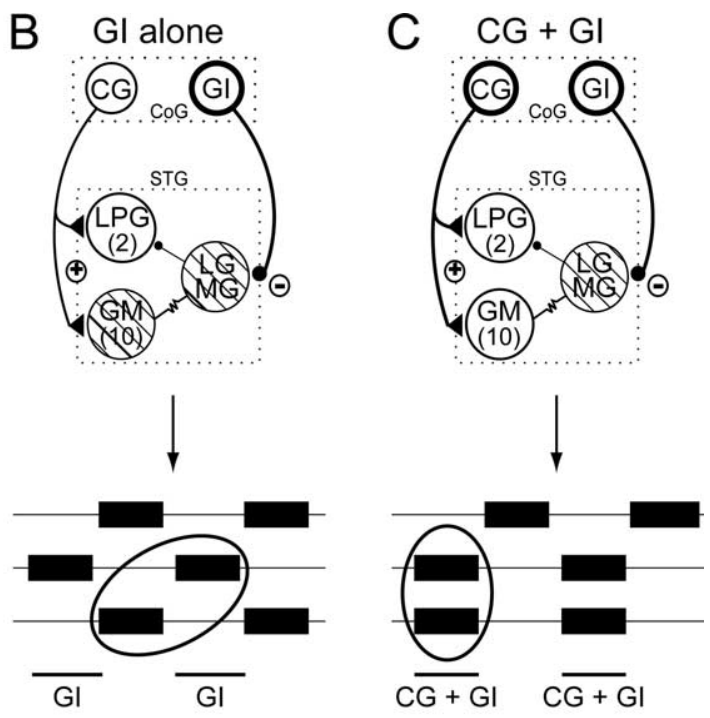

Pattern 2
Pattern 1

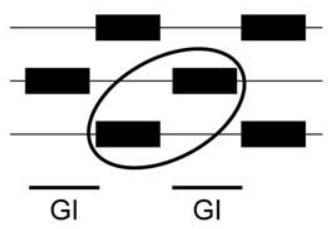

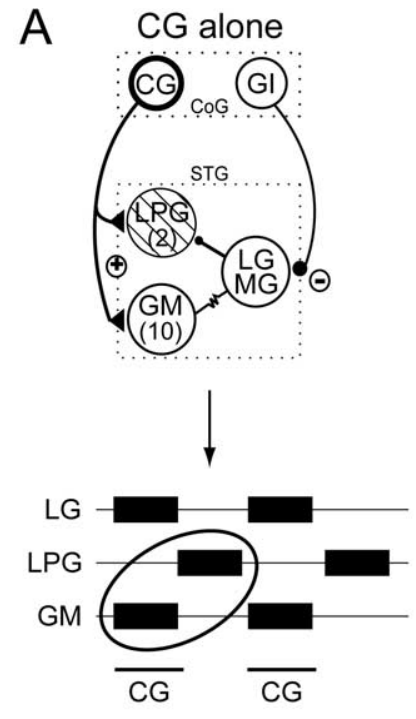

Figure 2. Two different gastric motor patterns elicited in vitro by cyclic activation of the CG and/or GI projection interneurons. Top schematics, Previously identified synaptic relationships between the C $G$ and Gl neurons and the gastric network (Combes et schematic denote motoneuron subtypes that are coactive with the rhythmically stimulated interneuron(s) (indicated with activation of a CG neuron alone generates a type 1 pattern (i.e., with LPG and GM neurons firing in phase opposition; ellipse). $B$ (1999a).

phase opposition to the LPG neurons (Fig. $2 A$, schematic). This pattern corresponds to a so-called "type 1 " gastric motor pattern, in which the neuron burst phase relationships remain similar to those expressed during spontaneous gastric network rhythmicity (Fig. $2 \mathrm{~A}$, bottom; compare with Fig. $1 C$ ).

In contrast to $\mathrm{CG}$, the second identified projection neuron (the GI interneuron) influences the gastric network via its direct inhibition of the LG-MG neuron pair. Rhythmic stimulation of the GI neuron thus hyperpolarizes and silences LG-MG and the electrically coupled GM neurons, and in the absence of LG-MG inhibition the spontaneously active LPG neurons fire in time with each GI stimulation (Fig. $2 B$ ). The motoneuron burst phase relationships in this GI-driven pattern therefore remain equivalent to the type 1 that occurs spontaneously or in response to $C G$ stimulation (Fig. 2, compare $B$ with $A$ ).

However, when the CG and GI interneurons are coordinately activated, the GI-mediated inhibition of the LG-MG circuit neurons causes coactivation of the GM and LPG neurons by CG excitatory input, and a completely different "type 2" gastric pattern, in which the LPG and GM motoneurons fire synchronously with the two projection neurons, but in antiphase with the LG-MG neurons (Fig. 2C). Therefore, the expression of the type 1 or type 2 versions of gastric circuit output in vitro, both of which have been observed in vivo (Combes et al., 1999a), depends on the relative levels of firing in the two parallel projection neuron pathways.

\section{The gastric mill network receives input from two different proprioceptor systems via the same projection neurons} Through its actions on the CG and GI interneurons, a previously identified proprioceptor, the AGR, whose bilateral stretchsensitive dendrites are activated by medial tooth protractor mus- 

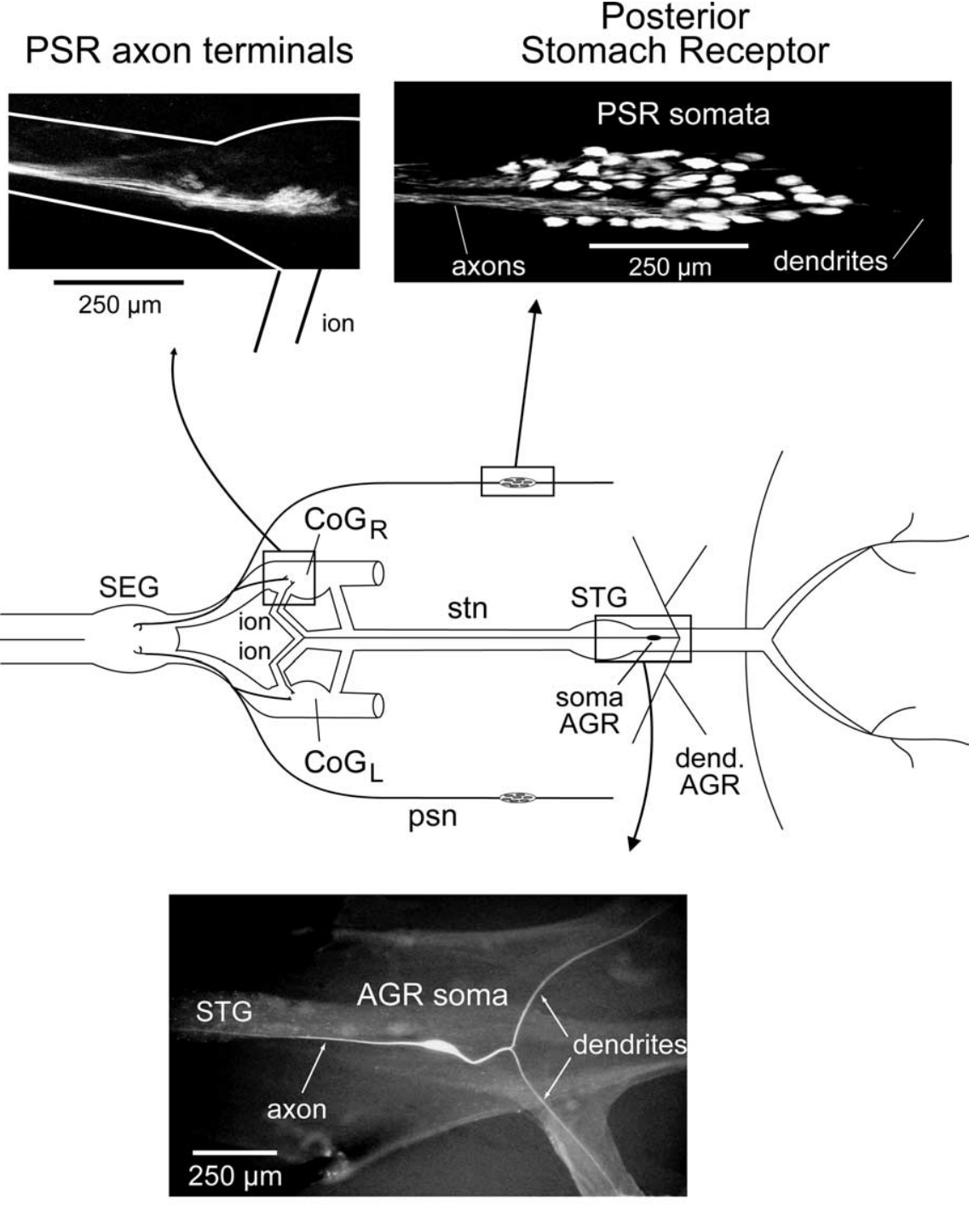

\section{Anterior Gastric Receptor}

Figure 3. Location and morphology of the PSR and the AGR in the STNS. Top left, Rhodamine-stained terminals of PSR axons in the ipsilateral COG (see STNS schematic). Branches of PSR axons also terminate in the SEG. Top right, Somata of PSR neurons in the distal region of the psn. Bottom, Lucifer-yellow-injected AGR showing its single bipolar soma, bilateral dendrites, and an ascending axon in the stn, which terminates in the bilateral $\mathrm{COGS}_{\text {. }} \mathrm{COG}_{\mathrm{L}}$, Left $\mathrm{COG}_{\mathrm{G}} \mathrm{COG}_{R}$, Right $\mathrm{CoG}$; dend, dendrite.

PSRs, also accesses the gastric mill network exclusively via the same CG and GI projection neurons as AGR. The PSR sensory system, originally identified by Dando and Laverack (1969), consists of bilaterally symmetric populations of stretchsensitive neurons whose somata are grouped in two peripheral ganglia located in the psn on either side of the stomach (Fig. 3, top; see also Fig. 1A). As seen from the rhodamine-dextran staining of Figure 3 (top right), each PSR is comprised of a population of 50-80 bipolar somata with axons that are conveyed centrally in the psn and terminate in the ipsilateral CoG (Fig. 3, top left) and the single SEG. Previous studies (Dando and Laverack, 1969; Nagy, 1981) and findings from in vivo recordings (our unpublished observations) have indicated that during spontaneous gastric mill movements, the receptor terminals of the two PSRs are activated by contraction of the lateral teeth opener muscles (gm3) that are innervated by the LPG motoneurons (Fig. 1C).

\section{Projection neuron-mediated effects of PSR on the gastric motor network} In contrast to AGR, PSR inhibits the CG interneurons. This is evident in the paired intracellular recordings in Figure $4 A$, in which moderate stimulation $(10 \mathrm{~Hz}$ for $5 \mathrm{~s}$ ) of PSR axons in a psn produced a hyperpolarization (bottom left trace) of the ipsilateral CG neuron via discrete IPSPs (bottom right) that followed each stimulus pulse 1:1 and at a constant delay. Conversely, and as for AGR, PSR depolarizes and excites the GI interneuron (Fig. 4A, top left) through discrete constant-latency EPSPs (Fig. $4 A$, top right) in response to the same psn stimulation. In both cases, these synaptic influences of PSR are probably direct, because they persisted under exposure to high divalent cation saline (data not shown). Moreover, no other

cle (gm1) contraction (Simmers and Moulins, 1988a,b; Combes et al., 1995), alters gastric network outputs as described above. AGR's bipolar soma is located caudally to the STG, and its axon projects in the stomatogastric nerve (stn) to the bilateral CoGs (Fig. 3, bottom), where it excites both the CG and GI interneurons. According to its rate of firing, AGR controls the balance of activation of the two projection neurons and thus determines the type of output pattern expressed by the downstream gastric mill network (Combes et al., 1999b). The higher intrinsic excitability of the CG neuron ensures that its influence on the gastric mill circuit predominates in response to low to moderate levels of AGR activation, thereby producing the type 1 gastric pattern (Fig. $2 A)$. During higher levels of receptor discharge, strong facilitation at the AGR-to-GI excitatory synapse leads to a coactivation of the two interneurons, resulting in a switch to the type 2 gastric pattern (Fig. 2C).

We now report that a different proprioceptor system, the
CoG interneuron has been found to be intercalated between the PSR and the gastric motor network, and consistent with this, the photoablation of both CG and GI cells in the same CoG (three preparations) by carboxyfluorescein injection and blue light illumination (Miller and Selverston, 1979) suppressed all actions of the ipsilateral PSR on the STG network (data not shown). These findings strongly argue that the action of each PSR on the STG gastric network, as demonstrated previously for AGR by similar photoinactivation experiments (Combes et al., 1999b), is mediated exclusively by the same two projection interneurons.

Because PSR synaptically excites only the GI neuron, we next tested whether stimulating a psn could elicit a type 1 gastric network pattern in a manner similar to that produced by direct experimental activation of GI, as in Figure $2 B$ (see also Combes et al., 1999a). This was assessed in the experiment of Figure $4 B$, in which a psn was cyclically stimulated $(10 \mathrm{~Hz}$ for $3 \mathrm{~s}$ every $10 \mathrm{~s})$ in 
a spontaneously nonrhythmic preparation. Within two psn train stimuli, a coordinated type 1 gastric rhythm was expressed in which the LPG neuron fired bursts in phase alternation with bursts in coactive GM (ellipse) and LG neurons. Moreover, in an already spontaneously rhythmic preparation, cyclic psn stimulation $(10 \mathrm{~Hz}$ for $5 \mathrm{~s}$ every $10 \mathrm{~s}$ ) was able to entrain ongoing gastric network activity (Fig. 4C). In this case, from the onset of psn stimulation, LPG neuron bursts were extended during each stimulus train, at which time the GM and LG neurons remained silent. Despite this cycleby-cycle prolongation of LPG discharge and the resultant slowing of the ongoing gastric rhythm, here again, the relative timing of bursts in the three different motoneuron types remained comparable with the burst pattern elicited by direct activation of the GI neuron alone (Fig. 2 B) (Combes et al., 1999a). These findings were therefore consistent with an action of PSR on the gastric mill network that was conveyed principally by the excitation of the GI neuron, a projection pathway influence that would be further reinforced by concomitant PSR inhibition of the CG interneuron (Fig. $4 A$, schematic).

\section{Combined effects of AGR and PSR on gastric network output}

Because a moderately activated AGR acts principally through its excitation of the CG interneuron (see above and Fig. $10 \mathrm{~A}$ ), whereas PSR excites GI, it was reasonable to predict that a conjoint activation of the two receptors should lead to a coactivation of the projection neurons, which in turn would elicit a switch in gastric circuit output to the type 2 pattern seen in Figure $2 C$. That this was indeed the case is illustrated in Figure 5, in which an intrasomatically recorded AGR was stimulated with repetitive depolarizing current pulses (for $4 \mathrm{~s}$ every $8 \mathrm{~s}$ ) to elicit rhythmic firing at moderate frequencies $(<20 \mathrm{~Hz})$ to drive a type $1 \mathrm{mo}-$ tor burst pattern (Fig. 5A1, left ellipse). In this typical AGR-evoked pattern, nerve-recorded GM motoneurons fired in phase with the activated receptor discharge, whereas the LPG motoneurons fired bursts in antiphase. This can be further seen at the beginning of the cycle-by-cycle plots in Figure $5 B$, in which the phases of successive GM and LPG burst onsets relative to the beginning of each AGR stimulation were measured in the recording sequence of Figure $5 \mathrm{~A}$. With AGR stimulation alone, the mean phase value for LPG burst onsets was $0.61 \pm 0.02$ (Fig. 5B, top dotted line), whereas that of GM bursts was $0.02 \pm 0.01$ (bottom dotted line). When a psn was additionally firing (ellipse).
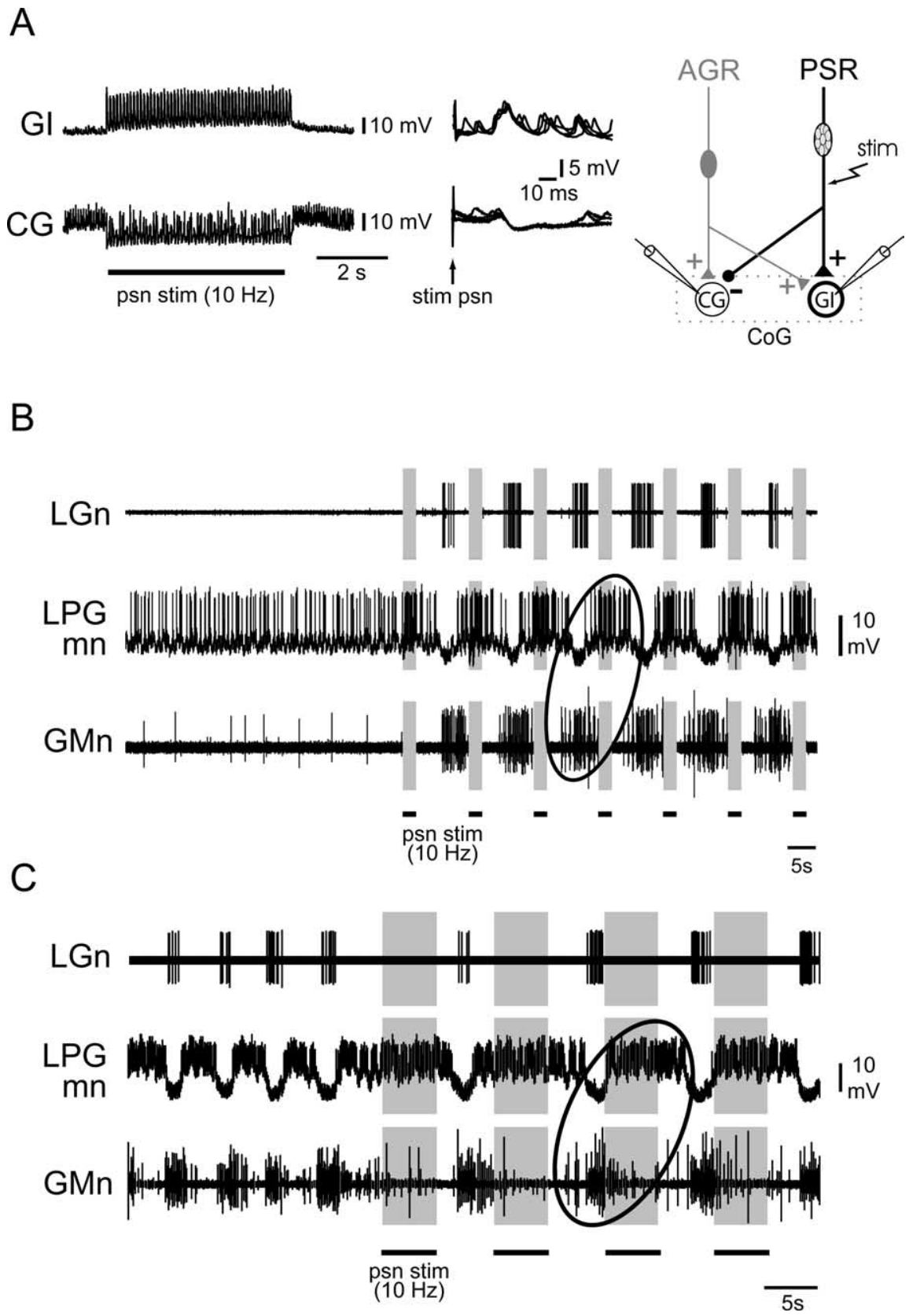

Figure 4. Effects of PSR stimulation (stim) on the $\mathrm{Gl}$ and $\mathrm{G}$ neurons and on gastric mill network activity. $A$, Stimulation ( $10 \mathrm{~Hz}$ for 5 s) of a psn excites an intracellularly recorded GI neuron via discrete, constant-latency EPSPs (see 5 superimposed sweeps at top right), whereas a simultaneously recorded $C G$ interneuron in the same $C O G$ was hyperpolarized by discrete IPSPs (see 5 superimposed sweeps at bottom right). These PSR synaptic influences are summarized in the schematic at right, which also includes (in gray) previously identified AGR inputs to the same projection neurons (Combes et al., 1999b). B, Cyclic psn stimulation ( $3 \mathrm{~s}$ train at $10 \mathrm{~Hz}$ every $10 \mathrm{~s}$ ) in the absence of spontaneous gastric rhythmicity elicits a type $1 \mathrm{gastric}$ motor pattern in which GM neurons recorded extracellularly in the corresponding motor nerves $(\mathrm{n})$ and an intracellularly recorded $L P G$ motoneuron $(\mathrm{mn})$ fire alternating bursts (ellipse). C, During spontaneous gastric network rhythmicity, cyclic psn stimulation ( $5 \mathrm{~s}$ trains at $10 \mathrm{~Hz}$ every 10 s) prolongs $L P G$ bursts and the ongoing gastric cycle period, and entrains the rhythm without altering $L P G$ and GM antiphase

stimulated $(10 \mathrm{~Hz}, 4 \mathrm{~s}$ every $8 \mathrm{~s})$ in synchrony with AGR, within two stimulus cycles the motor burst phase relations had changed to the type 2 pattern, in which LPG motoneurons now fired bursts simultaneously with GM (Fig. 5A1, right ellipse), resulting in similar phase values at $\sim 0$ (Fig. $5 B$ ). This pattern persisted throughout the paired receptor activation, but the burst phase relationships returned immediately to the type 1 version when psn stimulation ceased (Fig. 5A2,B). 


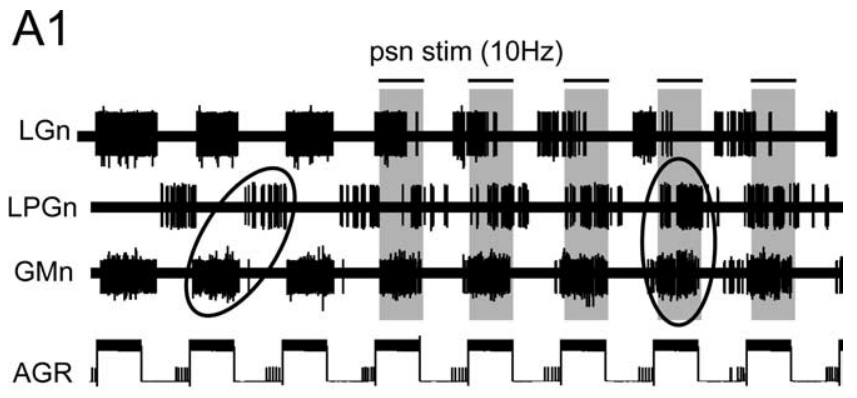

Pattern 1

Pattern 2
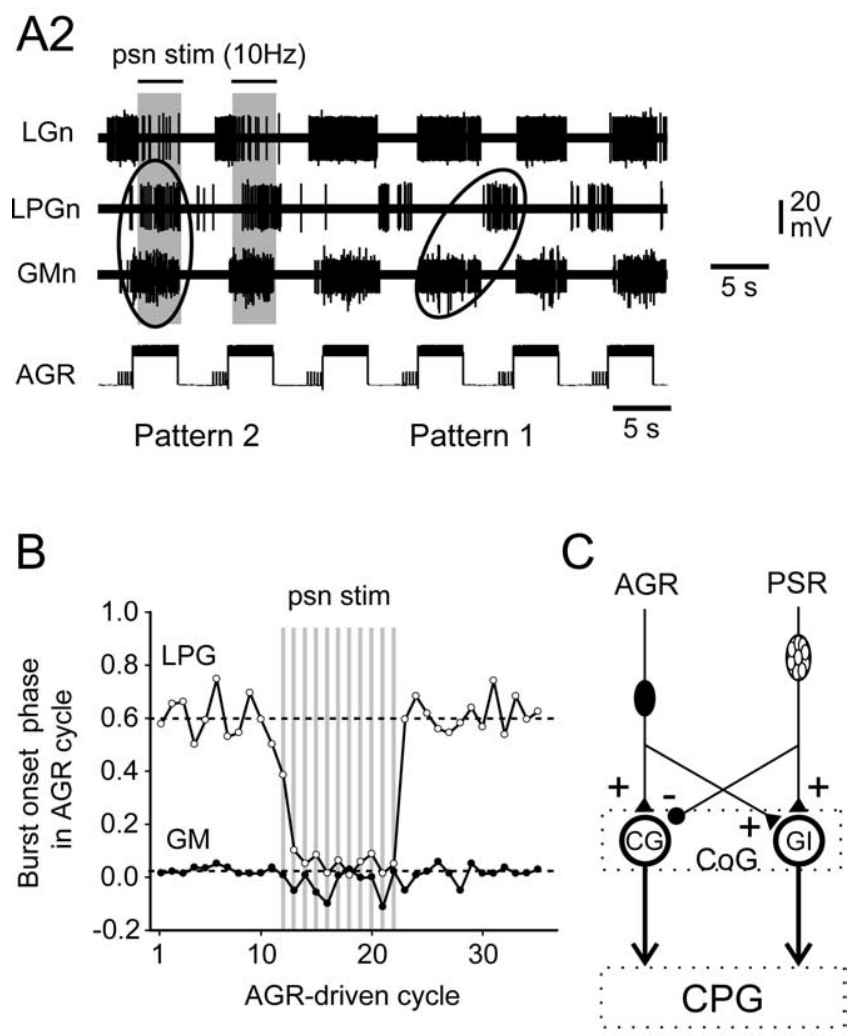

Pattern 2

Figure 5. Ability of combined PSR and AGR activation to elicit switching between different patterns of gastric mill output. A1, Cyclic stimulation of AGR by intrasomatic current injection elicited a type 1 gastric motor pattern [in which nerve (n)-recorded LPG and GM motoneurons fired in antiphase]. When psn was conjointly stimulated (indicated by bars above traces) with AGR, gastric activity switched to a type 2 pattern within two to three stimulus cycles. $\mathbf{A 2}$, Continuation of recordings in $\boldsymbol{A}$. When psn stimulation (stim) ceased, gastric activity driven by AGR alone returned immediately to the type 1 pattern. $B$, Analysis of the AGR + PSR stimulation sequence in $A$. Each vertically aligned pair of points indicates the phases of burst onset in the GM (filled circles) and LPG (open circles) motoneurons relative to AGR stimulation onset over 35 successive stimulus cycles. Additional rhythmic PSR stimulation ( $10 \mathrm{~Hz}, 8 \mathrm{~s}$ per cycle) is indicated by shaded vertical bars. Note the PSR-elicited switch in LPG/GM phase relationships from approximately phase opposition (pattern 1) to same phase (pattern 2). C, Summary schematic of the synaptic actions of costimulated AGR and PSR on the $C G$ and GI projection neurons, which lead to the interneurons being coactivated (in bold) and a resultant switch in motor burst phase relationships from a type 1 to a type 2 pattern.

These influences of combined receptor stimulation were therefore compatible with a coactivation of the two projection neurons via synaptic pathways summarized in Figure $5 C$, which specifically involved an AGR-elicited, CG-mediated excitation of the LPG and GM motoneurons in parallel with an
AGR/PSR-elicited, GI-mediated inhibition of the LG-MG neurons (as in Fig. 2C).

Prolonged influences of PSR on sensorimotor integration In addition to the transient effects of combined AGR and PSR on the gastric motor network, sustained actions of psn input, which could last for up to a minute or more, were evident at higher intensities of stimulation that again involved receptor influences on the GI and CG interneurons. As seen in the simultaneous intracellular recordings of the projection neurons in Figure $6 \mathrm{~A}$, and in accordance with Figure $4 A$, a single stimulus train (now at $20 \mathrm{~Hz}$ ) applied to the ipsilateral psn caused GI and CG to depolarize and hyperpolarize, respectively. However, after the termination of sensory nerve stimulation, the membrane potential (and firing frequencies) of the two projection neurons returned only slowly over the ensuing several seconds ( $12 \pm 5 s ; n=5$ trials in this experiment) to their original levels. As a consequence of this long-lasting PSR influence, the relative levels of spiking activity in the two interneurons, which was higher in CG than GI before psn stimulation, switched so that the GI interneuron now became the more active during and after the psn stimulation. In the example of Figure $6 A$, the control firing frequencies of CG and GI were 23 and $1.5 \mathrm{~Hz}$, respectively, whereas during the $2 \mathrm{~s}$ epoch immediately after the psn stimulation, the mean firing rate of CG was $15 \mathrm{~Hz}$, whereas that of GI remained at an elevated $45 \mathrm{~Hz}$.

How do these long-lasting PSR-derived influences alter the projection neurons' relationship with convergent input from AGR? This is assessed in the dual receptor stimulation experiment of Figure $6 \mathrm{~B}$, in which single-train stimulation $(20 \mathrm{~Hz}$ for $2 \mathrm{~s}$ ) of psn was interposed with ongoing cyclic activation of AGR by depolarizing current injection. Again, as in the different experiment of Figure $6 A$, the psn stimulation caused a prolonged synaptic excitation of GI and inhibition of CG (Fig. 6 B1). Before the sensory nerve stimulation, and as described previously (Combes et al., 1999b), moderate activation of AGR substantially increased the mean firing rate of the CG interneuron (here from 13 to $45.5 \mathrm{~Hz}$ ), whereas the GI interneuron was only weakly excited, with its firing rate increasing from 1 to $6 \mathrm{~Hz}$ (Fig. $6 \mathrm{B1}$, left rectangle). After the psn stimulation, however, the interneurons' responses to the same AGR activation were now superimposed on the sustained increased discharge of the psn-depolarized GI neuron and on the reduced activity of the psn-hyperpolarized CG (Fig. 6B1, right rectangle). This psn-derived modification in the sensitivity of the two projection neurons to AGR input is further evident in the spike frequency plots of Figure $6 B 2$, which show that the interneurons' altered responsiveness to AGR activation persisted for several tens of seconds after psn stimulation, with GI only returning to control levels of AGR-evoked discharge after a poststimulus interval of $>50 \mathrm{~s}$. Consequently, the ratio of GI and CG firing rates evoked by AGR activation (Fig. 6 B3), which was dominated by the CG interneuron before psn stimulation (GI/ $\mathrm{CG}=0.12 \pm 0.01)$, switched in favor of the GI neuron after psn stimulation and remained $>1$ for $\sim 10 \mathrm{~s}$ before returning to the prestimulus discharge ratio $\sim 40$ s later.

The downstream effect of this long-lasting psn-evoked change in the balance of projection neuron sensitivity to AGR input is seen in Figure 7, in which a single strong stimulation of a psn was interposed with ongoing cyclic activation of AGR while recording from the LG, LPG, and GM motoneurons in the corresponding motor nerves. Whereas before psn stimulation, moderate depolarizations of AGR elicited a type 1 gastric motor pattern, immediately after psn stimulation $(20 \mathrm{~Hz}$ for $4 \mathrm{~s})$, the continued acti- 
A

GI

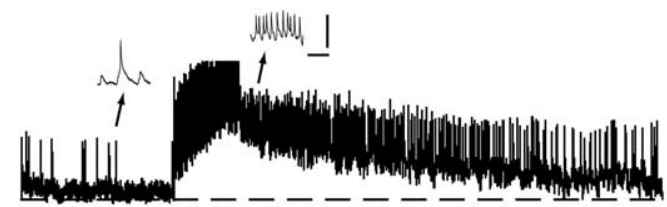

CG

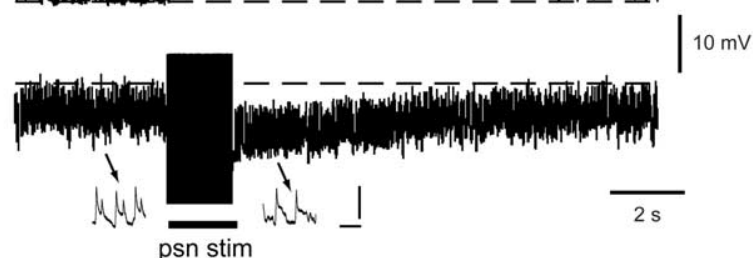

$(20 \mathrm{~Hz})$

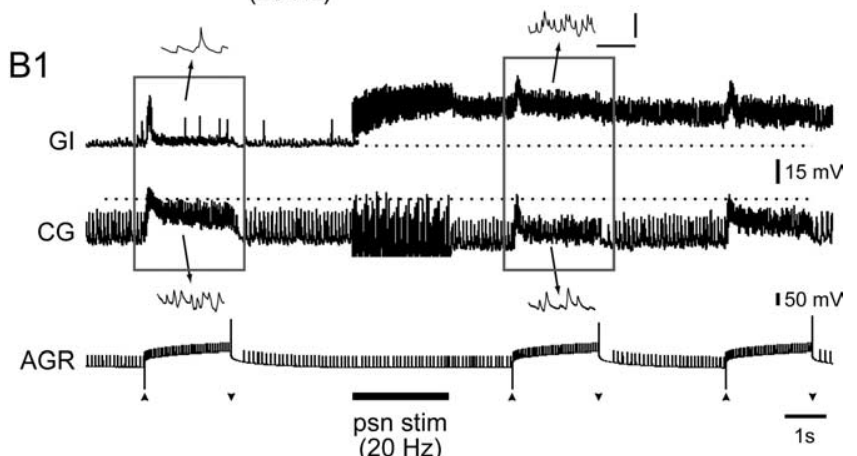

B2

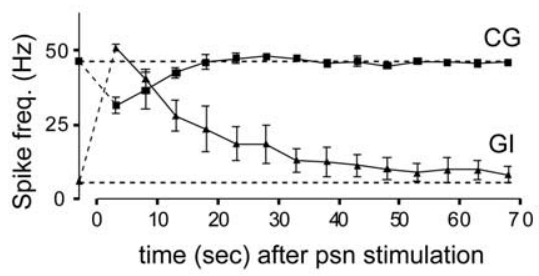

B3

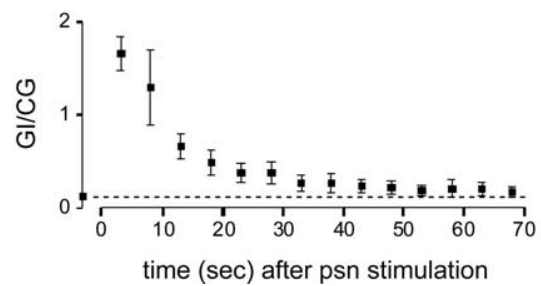

Figure 6. Long-lasting influences of PSR on the $\mathrm{Gl}$ and $\mathrm{CG}$ neurons, and the consequences for input from AGR.A, Prolonged synaptic depolarization and hyperpolarization, respectively, of intracellularly recorded $\mathrm{Gl}$ and $\mathrm{CG}$ neurons in response to a $2 \mathrm{~s}(2 \mathrm{~Hz})$ psn stimulation (stim). Insets, Expanded time-base samples from positions indicated on the main recordings. $\boldsymbol{B}$, psn stimulation-evoked changes in responsiveness of the two projection neurons to subsequent AGR activation. B1, Sample recordings from $\mathrm{Gl}$ and $\mathrm{CG}$ during $A G R$ activation (by depolarizing current injection between arrowheads) before and after a single-train $(20 \mathrm{~Hz})$ stimulation of psn. AGR's excitation of the interneurons is modulated according the long-lasting psn-evoked excitation and inhibition of $\mathrm{Gl}$ and $\mathrm{CG}$, respectively. B2, Mean spike frequency (freq.) of $\mathrm{Gl}$ and $\mathrm{CG}$ during successive AGR activations (as in B1) before and after psn stimulation. Before psn stimulation, the $C G$ neuron's mean firing frequency increased to $\sim 46 \mathrm{~Hz}$ (indicated by top dotted line) during AGRactivation, whereas GI was only weakly excited ( $\sim 6 \mathrm{~Hz}$, bottom dotted line). After psn stimulation, $C G$ was less activated by AGR over the ensuing $15 \mathrm{~s}$, whereas $\mathrm{Gl}$ was more sensitive to $A G R$ activation for $>50 \mathrm{~s}$. B3, As a result of these changes, the balance of firing between the two interneurons (Gl vs (G) switched from CG to Gl dominance after psn stimulation, with control discharge ratios returning after $\sim 45$ s. $\boldsymbol{A}, \boldsymbol{B} \mathbf{1}$, Insets, Calibration: $10 \mathrm{mV}, 100 \mathrm{~ms}$.

vation of AGR alone now evoked gastric motor bursts that had switched to the type 2 pattern. Furthermore, as with the prolonged effects of psn on the CG and GI neuron responses to AGR (Fig. 6B), this altered AGR-driven gastric pattern outlasted the original psn
A

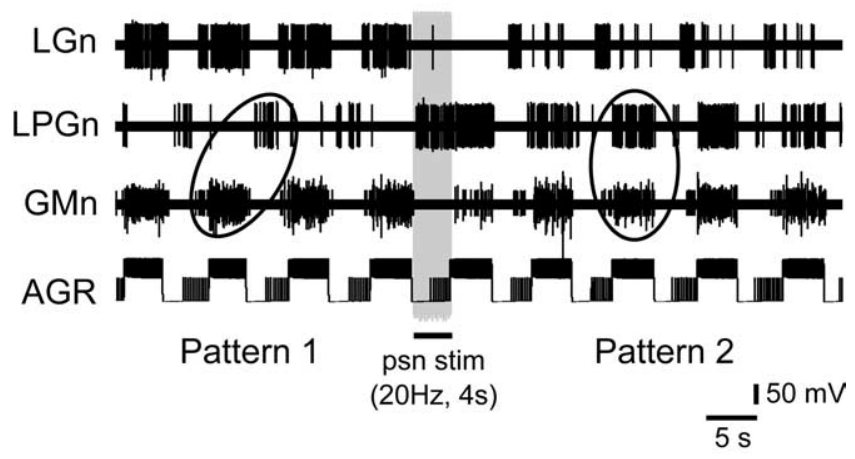

B

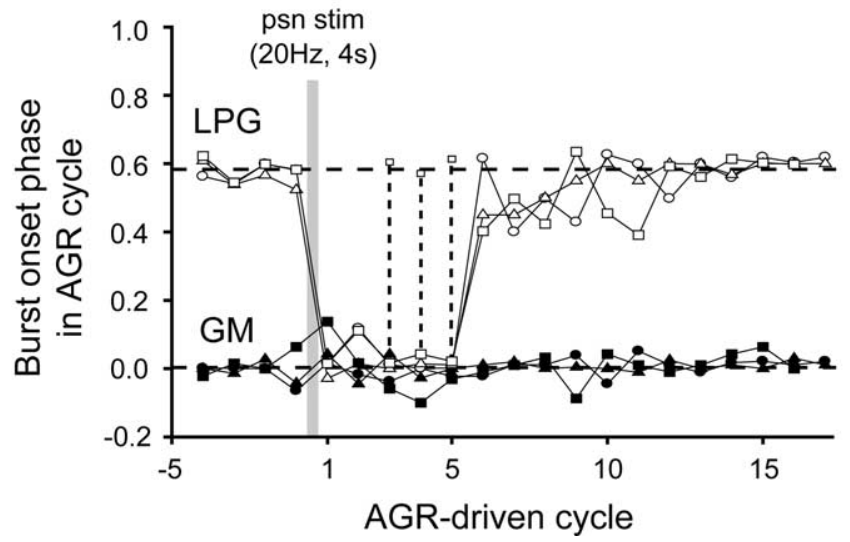

Figure 7. PSR can elicit a long-lasting modification of AGR's action on the gastric mill network. $A$, Before a single-train psn stimulation (stim), rhythmic AGR stimulation drives a type 1 gastric pattern (left ellipse), whereas after psn stimulation ( $20 \mathrm{~Hz}$ for $4 \mathrm{~s}$ ), ongoing AGR activation elicits for several cycles a type 2 burst pattern (right ellipse). $\boldsymbol{B}$, Time course of LPG and GM burst onset phase relationships with cyclic AGR stimulation before (cycles -5 to 0 ) and after (cycles 1-17) psn stimulation (gray bar). Data are from three trials in the same preparation as in A. Horizontal dotted lines indicate mean phase values for LPG (top) and GM (bottom) burst onsets during AGR stimulation alone. Data points for $L P G$ and GM pairs have the same symbol (open for LPG, filled for GM). Note that in one trial (squares and dashed vertical lines), in the post-psn stimulus period LPG fired transitional double bursts that were both in and out of phase with GM activity before control antiphase activity returned. $n$, Nerve.

stimulation by several tens of seconds (that included five motor pattern cycles in this trial) before gastric output returned to the original type 1 pattern that occurred before psn nerve stimulation. The analysis of three such trials is shown in Figure 7B, in which the phases of LPG and GM burst onsets were plotted over consecutive cycles of AGR stimulation. As seen in the raw data of Figure $7 \mathrm{~A}$, immediately after a single-train psn stimulation, the onsets of LPG bursts, which were previously in phase opposition (phase, $\sim 0.6$ ) with $\mathrm{GM}(\sim 0)$ in the activated AGR cycle, switched to coincident discharge with AGR for three to five poststimulus cycles before LPG returned to its control antiphase firing position over the ensuing three to seven cycles. Together, these results clearly indicate that PSR can modify AGR's influence on gastric circuit output, and that this switch is attributable to the ability of PSR, in both a transient and long-lasting manner, to change the excitability of the projection neurons through which input from the two receptors is transmitted to the motor network.

Direct PSR-to-AGR synaptic connections

In addition to their different synaptic actions on the CG and GI neurons, PSR and AGR interact upstream to the two projection neurons. Experimental support was found for two distinct types 


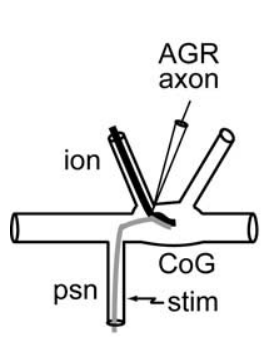

A1
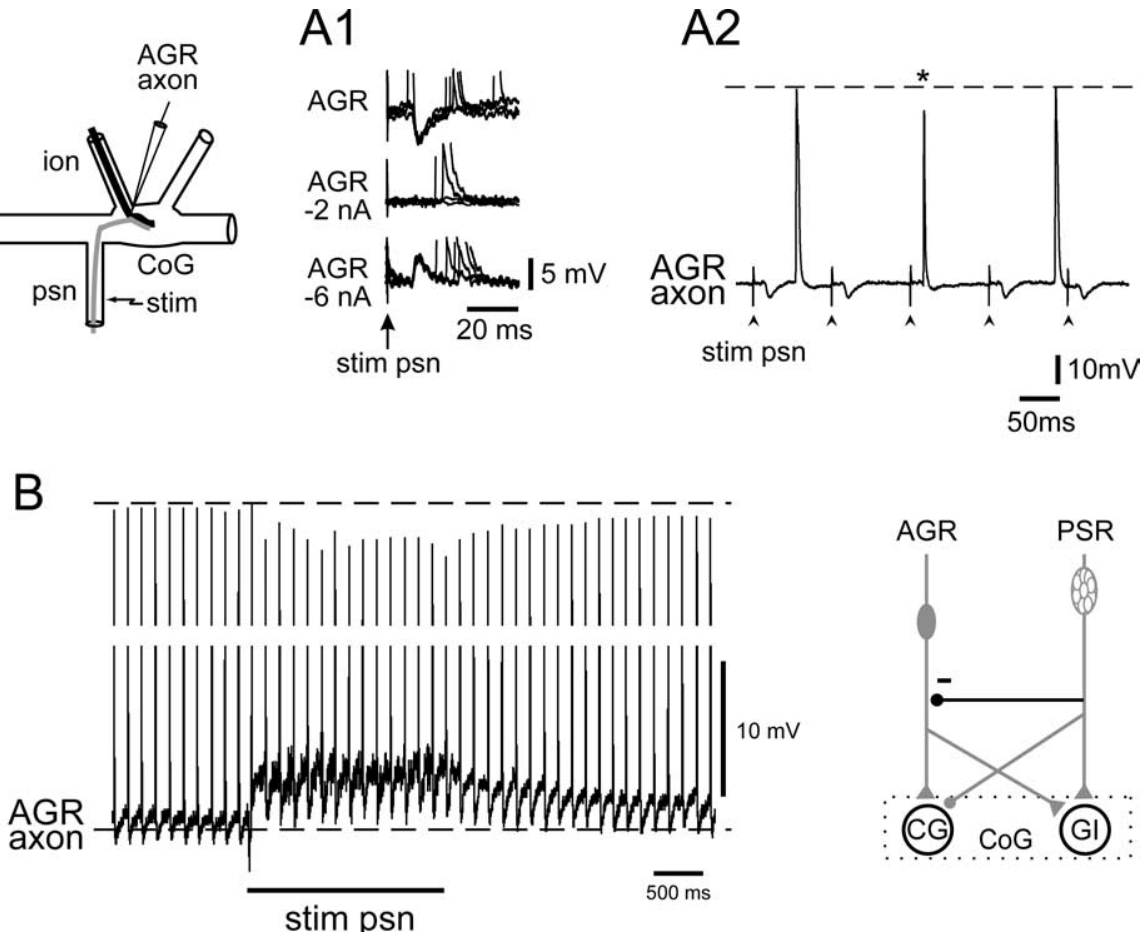

Figure 8. Transient synaptic and modulatory actions of PSR on AGR's axon terminals. $\boldsymbol{A}$, Intracellular recordings from near to AGR's axon terminals in a COG (see schematic) showing IPSPs occurring at constant latency after single-pulse psn stimulation (stim), which were diminished and then reversed by tonic AGR hyperpolarizing current injection (A1). $\boldsymbol{A 2}$, These psn-elicited IPSPs (by psn stimulation at arrowheads) attenuated spontaneous AGR when the IPSP and impulse coincided (asterisk). $\boldsymbol{B}$, Slower time-base recording showing that psn train stimulation $(20 \mathrm{~Hz}, 2 \mathrm{~s})$ caused a sustained membrane depolarization (compare with bottom dotted line) and attenuation of spontaneous spikes (top dotted line) in a recorded AGR axon terminal, with both effects outlasting the psn stimulation. The presynaptic inhibition of AGR by psn is indicated with a stick and ball symbol (in bold) in the schematic at bottom right.

of receptor-receptor influence, both of which would complement the PSR-derived influences on AGR input described above. These include a presynaptic inhibition of AGR's axon terminals by PSR and a selective modulatory enhancement of AGR's input to the GI interneuron.

Evidence that the PSR presynaptically inhibits AGR was obtained from intracellular recordings near the AGR's axon terminals in the CoG (Fig. $8 \mathrm{~A}$, schematic). As seen in Figure $8 \mathrm{Al}$ (top trace), single shock psn stimulation elicited a discrete hyperpolarizing IPSP in AGR's axon that followed each stimulation 1:1 and at constant latency. Continuous hyperpolarizing current applied to AGR suppressed (at $-2 \mathrm{nA}$ ) and then reversed (at -6 $\mathrm{nA}$ ) these IPSPs, confirming that they were mediated by chemical synaptic transmission. Moreover, the psn-derived IPSPs caused a decrease in both the size and duration of spontaneous spikes propagating into AGR's axon terminal when the two events occurred concurrently. This is evident in the different experiment of Figure $8 A 2$, in which the amplitude and duration of AGR axonal impulses arriving in the CoG were decreased significantly ( $p<0.01$; Mann-Whitney rank sum test) by $6.8 \pm 0.2 \mathrm{mV}$ $(\sim 10 \% ; n=20)$ and $5.7 \pm 0.4 \mathrm{~ms}(\sim 40 \% ; n=20)$, respectively, when they coincided with a PSR-evoked IPSP (see asterisk).

In addition to this coincidence-dependent attenuation of individual AGR impulses by PSR-elicited IPSPs, PSR also exerts effects on AGR's axon terminals that do not depend on the precise timing of receptor firing. As seen in the further experiment of Figure $8 \mathrm{~B}$, when a stimulus train (here $20 \mathrm{~Hz}$ for $2 \mathrm{~s}$ ) was applied to the ipsilateral psn, two additional synaptic responses were observable in AGR's axon terminal: (1) a tonic depolarization dur-

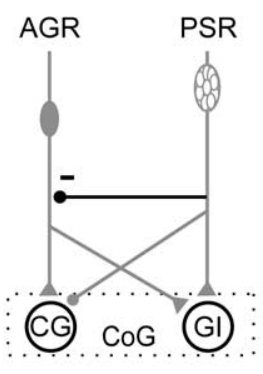

ing the psn stimulation associated with a significant $(p<0.01)$ attenuation in both amplitude (by $7.3 \pm 0.1$, i.e., $~ 9 \% ; n=$ 20) and duration (by $3.1 \pm 0.2 \mathrm{~ms}$, i.e., $\sim 25 \%$; $n=20$ ) of those spontaneous AGR impulses that did not coincide with discrete PSR-elicited IPSPs; and (2) a persistent membrane depolarization and spike height reduction after the termination of psn stimulation, with both AGR's membrane potential and impulse amplitude taking several seconds $(\sim 4 \mathrm{~s}$ in the case of Fig. $8 \mathrm{~B}$ ) to return to control levels. These data indicated a direct and slowly decaying depolarizing action of PSR on AGR in addition to transient spike-mediated synaptic inhibition, with both processes contributing to the reduction in size of action potentials propagating into the latter's presynaptic terminals. A likely consequence of these combined short- and longer-lasting presynaptic actions of PSR is that spike-evoked transmitter release by AGR is decreased, thereby diminishing AGR's ability to excite the postsynaptic CG and GI interneurons. Finally, it should be stressed that the presynaptic effects of PSR on AGR are likely to be underestimated in our recordings, because AGR's axon was impaled at its entrance into the CoG (Fig. 8 , top left schematic), presumably at some electrical distance from the actual location of PSR's output synapses within the ganglionic neuropil.

In contrast to the direct inhibitory actions of PSR on AGR, the excitatory synapse of AGR on GI is subject to selective upregulation by PSR. This further PSR influence is illustrated in Figure 9, in which AGR-evoked synaptic activity in a GI neuron was monitored intracellularly before and after a psn stimulation. As seen in Figure 9A, and in correspondence with the experiment in Figure $6 A$, a single-train stimulation of psn $(20 \mathrm{~Hz}$ for $10 \mathrm{~s})$ caused a slowly decaying depolarization of GI, which took $>40$ s to return to its control membrane potential in the poststimulus period. Concomitantly, and despite the prolonged depolarization of GI, the amplitudes of transient AGR spike-evoked synaptic potentials were also modulated by the psn stimulation, as is evident both in the data samples of Figure $9 B$, which shows superimposed AGR-spike triggered traces from the indicated positions in Figure $9 A$, and in the corresponding EPSP amplitude measurements of Figure 9C. In control conditions in this experiment, AGR impulses elicited EPSPs with mean amplitudes of $5 \pm 0.1 \mathrm{mV}$ in GI, but immediately after the psn stimulation, the AGR-evoked GI EPSPs had increased to $7 \pm 0.1 \mathrm{mV}$ and only returned to control amplitudes after a further $100 \mathrm{~s}$. This long-lasting potentiation of AGR's excitation of GI by PSR was not observed for AGR's excitatory synapse onto the CG interneuron (data not shown). Therefore, the selective enhancement of the AGR-to-GI synapse by PSR input would participate in further assisting the AGRmediated GI activation that is necessary for the prolonged switch to the type 2 gastric motor pattern seen in Figure 7. This increase in AGR's ability to excite the GI interneuron by PSR could be caused by a region-specific presynaptic modulation of neurotransmitter release from AGR's axon terminals or by a direct 

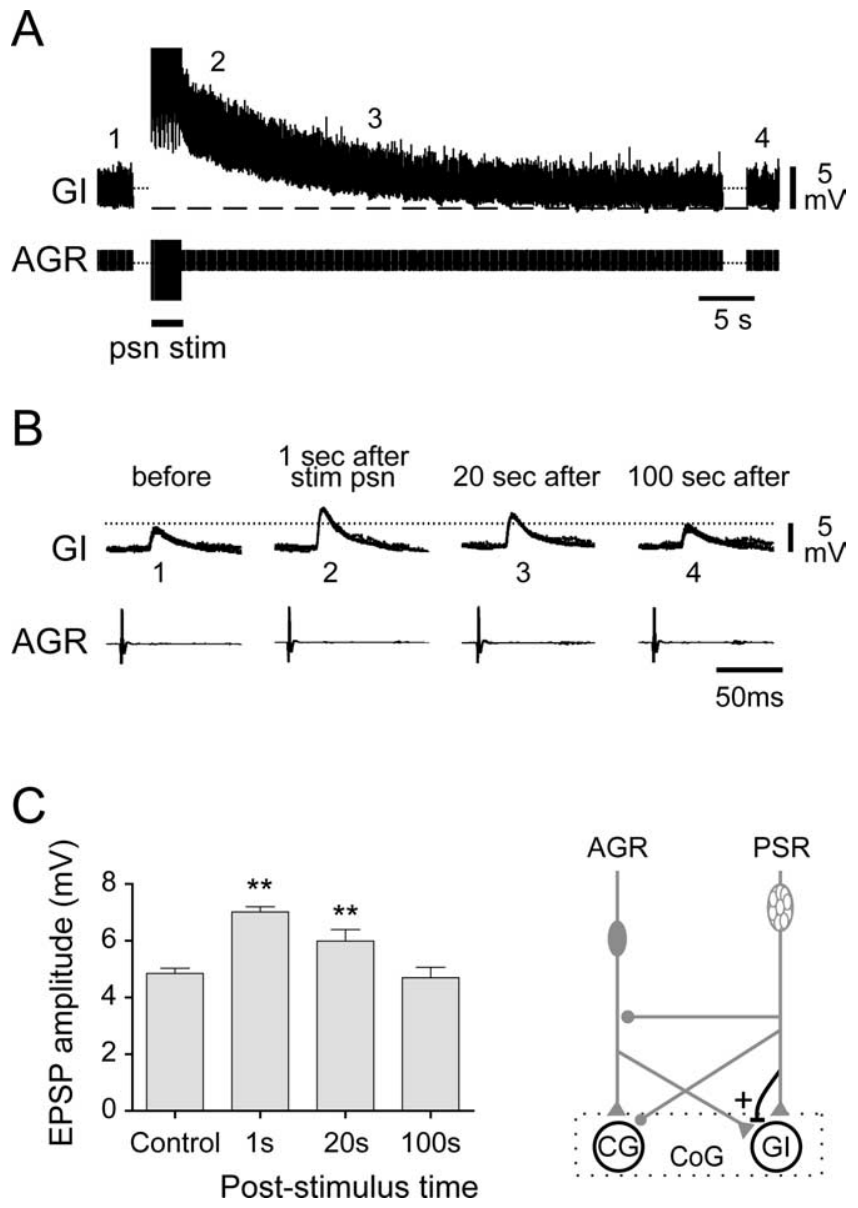

Figure 9. PSR causes long-lasting facilitation of AGR's excitatory input to the Gl interneuron. $A, \mathrm{~A} 10 \mathrm{~s}, 20 \mathrm{~Hz}$ psn stimulation (stim) elicited a slowly decaying membrane depolarization of an intracellularly recorded GI neuron. $\boldsymbol{B}$, Superimposed (5 sweeps per panel) AGR spike-evoked EPSPs (from recording in $A$, indicated by numbers) showing a long-lasting psn-induced increase in their amplitude. Note that the intracellular traces were aligned horizontally to the same baseline for amplitude comparison. C, Group analysis from three trials showing a significant increase $\left({ }^{* *} p<0.01\right.$, Dunnett's ANOVA posttest) in GI EPSP amplitude that lasted $>20$ s after psn stimulation. This facilitating action of PSR on the AGR-to-GI synapse is shown (in bold) in the schematic at bottom right.

action on the excitability of the postsynaptic GI neuron itself; we were unable to distinguish between these two possibilities because of the changes in GI membrane potential and conductances that PSR induces (Fig. $6 A, B$ ).

\section{Discussion}

The aim of this study was to explore the cellular and synaptic mechanisms involved in the integration of primary afferent information arising from two different proprioceptive systems that impinge on the same central motor network. To address this issue, we used the lobster stomatogastric nervous system, which has already been extensively described in terms of identified proprioceptive inputs (Simmers and Moulins, 1988a; Katz et al., 1989; Combes et al., 1995; Beenhakker et al., 2004, 2007), their interneuronal projection pathways, and target motor circuitry (Harris-Warrick and Katz, 1990; Marder and Calabrese, 1996; Combes et al., 1999a,b; Nusbaum and Beenhakker, 2002; Blitz et al., 2004). Here, we show that sensory input from one proprioceptor, the PSR, modifies the influence on the gastric mill network of another mechanoreceptor, AGR, via a combination of convergent postsynaptic actions on common projection inter-
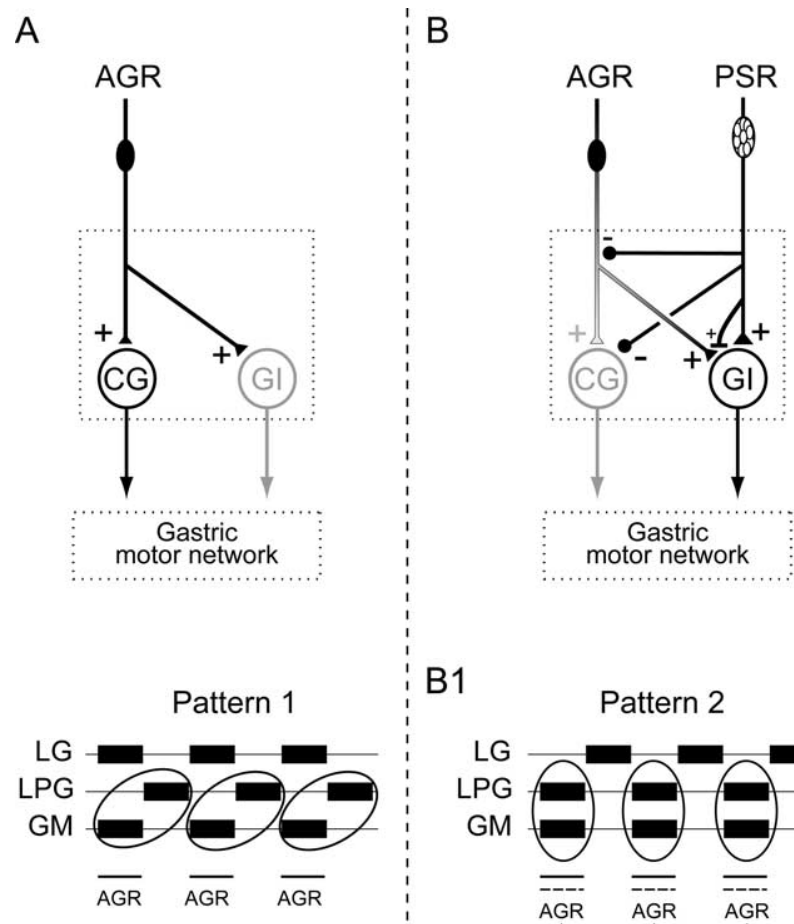

B1

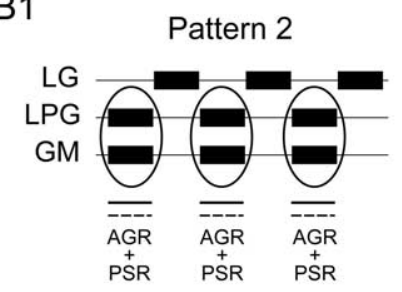

B2

Pattern 2

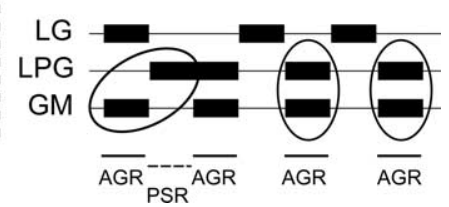

Figure 10. Summary of convergent proprioceptor-mediated regulation of gastric network activity. $\boldsymbol{A}$, Moderate AGR discharge drives a type 1 gastric pattern principally via excitation of the intercalated $(G$ projection neuron (see also Fig. $2 A$ ). B, Coincident AGR and PSR activity excites both the CG and $\mathrm{GI}$ projection neurons and elicits a type 2 gastric pattern (B1; see Fig. 5). Because of long-lasting actions of PSR at different presynaptic and postsynaptic loci, moderate AGR input after a recent PSR discharge leads to a prolonged switch to the type 2 gastric pattern (B2; see Fig. 7).

neurons and presynaptic interactions between two afferent pathways themselves. Figure 10 summarizes our results and shows the synaptic pathways recruited by AGR discharge alone (Fig. 10A) or in combination with PSR activation (Fig. $10 B$ ). Without PSR input, a moderately activated AGR influences the gastric motor circuit mainly through its excitation of the CG projection neuron (Fig. 10A), which in turn drives gastric network output in a type 1 pattern in which the lateral teeth (LPG) and medial tooth (GM) power-stroke motoneurons fire in phase opposition (see also Fig. $2 A$ ). During coincident AGR and PSR activation (Fig. 10 B1) or after elevated discharge in the latter (Fig. 10 B2), the same stimulation of AGR elicits a switch in gastric circuit output to the type 2 pattern, in which LPG and GM motoneurons fire coordinately.

We have identified several complementary presynaptic and postsynaptic, short- and long-lasting PSR actions that alter AGR's influence on the gastric motor network. First, PSR presynaptically inhibits AGR's axon terminals both by transient hyperpolarizing IPSPs and by slowly decaying depolarizing potentials, which together result in a reduced ability of the terminal membrane to support action potentials. Second, PSR partially com- 
pensates for the likely resulting decrease in synaptic effectiveness by potentiating AGR's excitation of the GI interneuron but not of CG. Third, the biasing of information transfer from AGR through GI is further reinforced by long-lasting PSR influences on the excitability of the postsynaptic projection neurons themselves, involving a prolonged synaptic excitation and inhibition of GI and CG, respectively.

Pivotal to these integrative processes is that inputs from the two receptors are carried to the gastric motor circuit via the same two projection neurons. Whereas PSR reaches the gastric network through its excitation of the inhibitory GI interneuron (excitatory CG being inhibited by the receptor), AGR's input to the motor circuit is mediated either by excitation of CG alone, or through a coactivation of both interneurons (Combes et al., 1999a). It is well known that the same premotor interneurons can be targeted by different sensory systems (Lundberg, 1979; Jankowska, 1992; Burrows and Laurent, 1993; Hultborn, 2006; Hultborn and Nielsen, 2007). For example, a facilitatory interaction between hindlimb muscle spindle and cutaneous afferents resulting from their convergence onto common interneurons has been described in cat (Kirkwood et al., 1987). Inputs from joint receptors (Lundberg et al., 1978) and spindle afferents (Jankowska and McCrea, 1983) also influence tension regulation from Golgi tendon organs, again through convergence at the premotoneuronal level. In locust, mechanosensory afferents from different leg exteroceptors make central connections with the same local interneurons (Burrows and Newland, 1994), and in the stomatogastric system of crabs, mechanoreceptors other than PSR and AGR also have access to motor circuitry via common projection neurons (Blitz et al., 2004; Beenhakker et al., 2007).

In all cases, the convergence of sensory information at the premotor level is thought to provide an integrating locus at which facilitating or competing input signals are summed or subtracted to maximize the production of appropriate motor responses (Burrows and Newland, 1994). However, although functionally different sensory systems may share the same or overlapping subpopulations of projection neurons, they can still elicit distinct patterns of motor output when activated independently (Deliagina et al., 2002; Blitz et al., 2004). Alternatively, two distinct proprioceptors may separately exert similar effects on a target motor network by coactivating the same projection neurons, but one proprioceptor can occlude the excitatory actions of the other when the two sensory systems are conjointly active (Beenhakker et al., 2007).

In our model, despite different actions on the same projection neurons, two distinct mechanoreceptors are able independently to exert qualitatively similar influences on gastric network output, but when activated simultaneously or consecutively, they produce a switch to a completely different output pattern. This novel convergent action resides with the ability of one proprioceptor (PSR) to select the intervening projection neuron pathway through which sensory information from the second receptor (AGR) is conveyed to downstream motor circuitry. It is interesting that the rechanneling of sensory signals to motor circuitry has already been documented in vertebrates, in which the feedback control of voluntary limb movements depends on multisensory convergence onto parallel interneurons that provide alternative reflex pathways to motoneurons and the production of differing forms of movement (Hultborn, 2001). Here, however, the routing of sensory information through these interneuronal pathways is thought to be specified by descending supraspinal commands.

An important substrate for the biasing of AGR's input to its projection neuron targets resides with PSR's ability to presynaptically inhibit AGR's axon terminals. This direct PSR-to-AGR connection evidently engages two distinct inhibitory mechanisms: conventional synaptic inhibition and a slowly decaying depolarization. Both processes, for which precedents have been well described in the literature (Frost et al., 1985; Kretz et al., 1986; Clarac and Cattaert, 1996; Nusbaum et al., 1997; Rudomin, 2002), constitute mechanisms for shunting or inactivating inward currents giving rise to presynaptic impulses, thereby diminishing their size/duration with a resultant reduction in transmitter release (Cattaert and El Manira, 1999). Unfortunately, because input from PSR also exerts direct postsynaptic actions on CG and GI, we were unable to establish whether the PSR-derived presynaptic effects on AGR did indeed lead to a reduced influence of the latter on the projection neurons.

Presynaptic inhibition resulting from convergent sensorysensory interactions has been documented in other invertebrate motor systems, including central connections between afferent terminals arising from the same insect leg proprioceptor (Burrows and Laurent, 1993; Burrows and Matheson, 1994), or from functionally distinct limb sense organs (Stein and Schmitz, 1999). This inhibition is thought to serve as a gain control mechanism that prevents saturation of postsynaptic responses and ensures the appropriate tuning of posture and movement. The use of presynaptic inhibition by one proprioceptor to bias another's access to motor circuitry has been recently reported in the gastric mill system of crabs (Beenhakker et al., 2007). Here, however, and notwithstanding the possibility of additional direct sensory-sensory interactions similar to those found in our study, the site identified for receptor-mediated presynaptic inhibition is located in the downstream axon terminals of postsynaptic projection neurons rather than in primary afferent terminals (see also Beenhakker et al., 2005).

In parallel with PSR's ability to presynaptically inhibit AGR, PSR facilitates AGR's activation of the GI neuron, thereby offsetting the reduction in AGR's excitatory drive to both the CG and GI projection neurons. We do not know whether this additional PSR action at the AGR-GI synapse, which appears to involve a modulatory process, derives from a selective enhancement of transmitter release from AGR's presynaptic terminals with GI and/or from an increase in postsynaptic responsiveness. Although distinguishing experimentally between these two possibilities is again rendered difficult because of PSR's concomitant excitation of GI, clearly such influences acting in combination with PSR-mediated inhibition of CG would ensure that incoming mechanosensory signals from AGR are routed preferentially to the gastric motor network via the GI interneuron. Furthermore, the involvement of PSR modulatory actions in this biasing process provides a mechanism by which the two proprioceptors may interact centrally in a manner that extends over several cycles of gastric motor activity and is therefore relatively independent of the timing of peripheral receptor activation.

The present work thus adds to our understanding of the way in which distinct sensory systems are able to regulate motor network activity, and in particular, points to the extent to which convergent afferent signaling is processed at first-order synapses in sensorimotor pathways. Although studies on a variety of systems (Clarac and Cattaert, 1996; Parker and Grillner, 1996; El Manira et al., 1997; Nusbaum et al., 1997; Rudomin, 2002; Frost et al., 2003), including the crustacean stomatogastric system (Beenhakker et al., 2005, 2007), have identified integrative roles for various mechanisms at primary afferent synapses, how such multiple processes enable converging input pathways to direct their information to downstream rhythmically active motor circuitry has thus far not been described. It should also be noted that 
unlike the solitary AGR neuron, PSR is a multicellular stretch receptor whose dendrites terminate in the stomach wall (Dando and Laverack, 1969). It is therefore possible that the physiological activation of PSR is not equivalent in all its component cells, but that they are recruited differentially according to muscle movement and/or stomach distention. It is possible, therefore, that biologically relevant activation of PSR, rather than en bloc nerve stimulation as used in our in vitro experiments, will reveal an even wider range of integrative processes than those described here.

\section{References}

Beenhakker MP, Blitz DM, Nusbaum MP (2004) Long-lasting activation of rhythmic neuronal activity by a novel mechanosensory system in the crustacean stomatogastric nervous system. J Neurophysiol 91:78-91.

Beenhakker MP, DeLong ND, Saideman SR, Nadim F, Nusbaum MP (2005) Proprioceptor regulation of motor circuit activity by presynaptic inhibition of a modulatory projection neuron. J Neurosci 25:8794-8806.

Beenhakker MP, Kirby MS, Nusbaum MP (2007) Mechanosensory gating of proprioceptor input to modulatory projection neurons. J Neurosci 27:14308-14316.

Blitz DM, Beenhakker MP, Nusbaum MP (2004) Different sensory systems share projection neurons but elicit distinct motor patterns. J Neurosci 24:11381-11390.

Burrows M, Laurent G (1993) Synaptic potentials in the central terminals of locust proprioceptive afferents generated by other afferents from the same sense organ. J Neurosci 13:808-819.

Burrows M, Matheson T (1994) A presynaptic gain control mechanism among sensory neurons of a locust leg proprioceptor. J Neurosci $14: 272-282$.

Burrows M, Newland PL (1994) Convergence of mechanosensory afferents from different classes of exteroceptors onto spiking local interneurons in the locust. J Neurosci 14:3341-3350.

Büschges A (2005) Sensory control and organization of neural networks mediating coordination of multisegmental organs for locomotion. J Neurophysiol 93:1127-1135.

Cattaert D, El Manira A (1999) Shunting versus inactivation: analysis of presynaptic inhibitory mechanisms in primary afferents of the crayfish. J Neurosci 19:6079-6089.

Clarac F, Cattaert D (1996) Invertebrate presynaptic inhibition and motor control. Exp Brain Res 112:163-180.

Combes D, Simmers J, Nonnotte L, Moulins M (1993) Tetrodotoxinsensitive dendritic spiking and control of axonal firing in a lobster mechanoreceptor neurone. J Physiol 460:581-602.

Combes D, Simmers J, Moulins M (1995) Structural and functional characterization of a muscle tendon proprioceptor in lobster. J Comp Neurol 363:221-234

Combes D, Meyrand P, Simmers J (1999a) Motor pattern specification by dual descending pathways to a lobster rhythm-generating network. J Neurosci 19:3610-3619.

Combes D, Meyrand P, Simmers J (1999b) Dynamic restructuring of a rhythmic motor program by a single mechanoreceptor neuron in lobster. J Neurosci 19:3620-3628.

Dando MR, Laverack MS (1969) The anatomy and physiology of the posterior stomach nerve (p.s.n.) in some decapod crustacea. Proc R Soc Lond B Biol Sci 171:465-482.

Deliagina TG, Zelenin PV, Orlovsky GN (2002) Encoding and decoding of reticulospinal commands. Brain Res Rev 40:166-177.

El Manira A, Tegnér J, Grillner S (1997) Locomotor-related presynaptic modulation of primary afferents in the lamprey. Eur J Neurosci 9:696-705.

Frost WN, Castellucci VF, Hawkins RD, Kandel ER (1985) Monosynaptic connections made by the sensory neurons of the gill- and siphonwithdrawal reflex in Aplysia participate in the storage of long-term memory for sensitization. Proc Natl Acad Sci U S A 82:8266-8269.

Frost WN, Tian LM, Hoppe TA, Mongeluzi DL, Wang J (2003) A cellular mechanism for prepulse inhibition. Neuron 40:991-1001.

Grillner S, Wallén P (2002) Cellular bases of a vertebrate locomotor systemsteering, intersegmental and segmental co-ordination and sensory control. Brain Res Rev 40:92-106.

Harris-Warrick RM, Katz PS (1990) Sensory modulation of motor pattern generators in the crab stomatogastric ganglion. In: Crustacean neurobi- ology (Wiese KW, Krenz W-D, Tautz J, Reichert H, Mulloney B, eds), pp 431-438. Basel: Birkhauser Verlag.

Hooper SL, Moulins M, Nonnotte L (1990) Sensory input induces longlasting changes in the output of the lobster pyloric network. J Neurophysiol 64:1555-1573.

Hultborn H (2001) State-dependent modulation of sensory feedback. J Physiol 533:5-13.

Hultborn H (2006) Spinal reflexes, mechanisms and concepts: from Eccles to Lundberg and beyond. Prog Neurobiol 78:215-232.

Hultborn H, Nielsen JB (2007) Spinal control of locomotion-from cat to man. Acta Physiol 189:111-121.

Jankowska E (1992) Interneuronal relay in spinal pathways from proprioceptors. Prog Neurobiol 38:335-378.

Jankowska E, McCrea DA (1983) Shared reflex pathways from Ib tendon organ afferents and Ia muscle spindle afferents in the cat. J Physiol 338:99-111.

Katz PS, Harris-Warrick RM (1990) Neuromodulation of the crab pyloric central pattern generator by serotonergic/cholinergic proprioceptive afferents. J Neurosci 10:1495-1512.

Katz PS, Eigg MH, Harris-Warrick RM (1989) Serotonergic/cholinergic muscle receptor cells in the crab stomatogastric nervous system. I. Identification and characterization of the gastropyloric receptor cells. J Neurophysiol 62:558-570.

Kirkwood PA, Schomburg ED, Steffens H (1987) Facilitatory interaction in spinal reflex pathways from nociceptive cutaneous afferents and identified secondary spindle afferents in the cat. Exp Brain Res 68:657-660.

Kretz R, Shapiro E, Kandel ER (1986) Presynaptic inhibition produced by an identified presynaptic inhibitory neuron. I. Physiological mechanisms. J Neurophysiol 55:113-130.

Lundberg A (1979) Multisensory control of spinal reflex pathways. Prog Brain Res 50:11-28.

Lundberg A, Malmgren K, Schomburg ED (1978) Role of joint afferents in motor control exemplified by effects on reflex pathways from Ib afferents. J Physiol 284:327-343.

Marder E, Calabrese RL (1996) Principles of rhythmic motor pattern generation. Physiol Rev 76:687-717.

Miller JP, Selverston A (1979) Rapid killing of single neurons by irradiation of intracellularly injected dye. Science 206:702-704.

Nagy F (1981) Etude del'expression d'activités motrices rythmiques organisées par des générateurs paucineuroniques du système nerveux stomatogastrique des crustacés décapodes. Thèse d'Etat, Université Bordeaux 1.

Nargeot R, Moulins M (1997) Sensory-induced plasticity of motor pattern selection in the lobster stomatogastric nervous system. Eur J Neurosci 9:1636-1645.

Nusbaum MP, Beenhakker MP (2002) A small-systems approach to motor pattern generation. Nature 417:343-350.

Nusbaum MP, El Manira A, Gossard JP, Rossignol S (1997) Presynaptic mechanisms during rhythmic activity in vertebrates and invertebrates. In: Neurons, networks and motor behavior (Stein PSG, Grillner S, Selverston AI, Stuart DG, eds), pp 237-253. Cambridge: MIT.

Parker D, Grillner S (1996) Tachykinin-mediated modulation of sensory neurons, interneurons, and synaptic transmission in the lamprey spinal cord. J Neurophysiol 76:4031-4039.

Pearson KG (1995) Proprioceptive regulation of locomotion. Curr Opin Neurobiol 5:786-791.

Rossignol S, Dubuc R, Gossard JP (2006) Dynamic sensorimotor interactions in locomotion. Physiol Rev 86:89-154.

Rudomin P (2002) Selectivity of the central control of sensory information in the mammalian spinal cord. Adv Exp Med Biol 508:157-170.

Selverston AI, Moulins M (1987) The crustacean stomatogastric system. Heidelberg: Springer.

Simmers J, Moulins M (1988a) A disynaptic sensorimotor pathway in the lobster stomatogastric system. J Neurophysiol 59:740-756.

Simmers J, Moulins M (1988b) Nonlinear interneuronal properties underlie integrative flexibility in a lobster disynaptic sensorimotor pathway. J Neurophysiol 59:757-777.

Soffe SR (1997) The pattern of sensory discharge can determine the motor response in young Xenopus tadpoles. J Comp Physiol [A] 180:711-715.

Stein W, Schmitz J (1999) Multimodal convergence of presynaptic afferent inhibition in insect proprioceptors. J Neurophysiol 82:512-514.

Yakovenko S, McCrea DA, Stecina K, Prochazka A (2005) Control of locomotor cycle durations. J Neurophysiol 94:1057-1065. 